\title{
Early detection and primary prevention system in COVID-19 transmission to staff and residents in the department of obstetrics and gynecology
}

Muhammad Nurhadi Rahman,* Detty Siti Nurdiati, Nuring Pangastuti, Anis Widyasari, Fauzan Achmad Maliki, Sarrah Ayu Andari, and Khairina Hashifah

Department of Obstetrics and Gynecology, Faculty of Medicine, Public Health, and Nursing, Universitas Gadjah Mada, Yogyakarta, Indonesia

\section{KEYWORDS}

Schedule arrangement

Personal protective equipment

Contact tracing

Early detection
ABSTRACT COVID-19 is currently a global pandemic because of its fast transmission in humans. The disease can be symptomatic or asymptomatic. Patients with the disease can transmit the infection to the medical team who are exposed without proper protection. Prevention of transmission is our main focus of attention as well as early recognition of exposed medical teams among obstetrics and gynecology residents and consultants in our hospital. This article aimed to describe the COVID-19 early detection and primary prevention system among staff and residents in the Obstetrics and Gynecology (OBSGYN) Department, Faculty of Medicine, Public Health and Nursing, Universitas Gadjah Mada and Dr. Sardjito General Hospital from April to June 2020. The data were acquired from the reports of the COVID-19 taskforce from the OBSGYN Department. In order to prevent the transmission of COVID-19 infection, actions taken by the OBSGYN Department are as follows: a) arrangement of staff and residents watch schedules, b) usage of PPE (Personal Protective Equipment) according to protocol, c) contact tracing, and d) early detection. Resident watch schedule arrangement is done by withdrawing residents from affiliated hospitals so that the division of daily duty schedules at Dr. Sardjito General Hospital is divided into 2 shifts of 12 hours each. The PPE usage policy is adjusted according to the risk of COVID-19 exposure in different environments and in accordance with established standards. A total of 65 out of 74 residents (88\%) had been exposed to suspected COVID-19 cases, and among the exposed residents, 12 residents $(18 \%)$ were self-isolated because of the occurrence of symptoms, exposure to patients with positive COVID-19 rapid antibody test, or exposure to patients with positive COVID-19 Polymerase Chain Reaction (PCR) test. The department also conducted early detection of COVID-19 infection among all staff members and residents. As many as 13 out of 27 staff (48\%) underwent COVID-19 rapid antibody test with $100 \%$ negative results, 66 of the 74 residents (89\%) also underwent COVID-19 rapid antibody test with $100 \%$ negative results. In conclusion, the OBSGYN Department has implemented several measures to prevent transmission of COVID-19 among its staff and residents with adequate satisfactory results.

(c) The Journal 2021. This article is distributed under a Creative Commons Attribution-ShareAlike 4.0 International license.

\section{Introduction}

Coronavirus is a large family of viruses that cause illness ranging from mild to severe symptoms. There are at least two types of coronavirus that are known to cause diseases that can cause severe symptoms such as Middle East Respiratory Syndrome (MERS) and Severe Acute Respiratory Syndrome (SARS). Coronavirus Disease 2019 (COVID-19) is a new type of disease that has never been previously identified

*Correspondence: adirrahman@ymail.com

Department of Obstetrics and Gynecology, Faculty of Medicine, Public Health and Nursing, Universitas Gadjah Mada, J. Farmako, Sekip Utara, Yogyakarta 55281, Indonesia in humans. The virus that causes COVID-19 is called SARS-CoV-2. Corona viruses are zoonotic, i.e. they are transmitted between animals and humans. ${ }^{1}$ Previous research found that SARS is transmitted from civets to humans while MERS is transmitted from camels to humans. Meanwhile, the animal which is the source of transmission of COVID-19 is still unknown. ${ }^{2}$

Common signs and symptoms of COVID-19 infection include acute respiratory symptoms such as fever, cough, and shortness of breath. The average incubation period is 5-6 days with the longest incubation period of 14 days. Severe cases of COVID-19 can cause pneumonia, acute respiratory 
distress syndrome, kidney failure, and even mortality. The clinical signs and symptoms reported in the majority of cases are fever, with some cases reporting shortness of breath, and chest $x$-rays showing large pneumonia infiltrates in both lungs. ${ }^{3}$

Based on scientific evidence, COVID-19 can be transmitted from person to person through close contact and droplets. People who have the highest risk of contracting this disease are people who have close contact with COVID-19 patients, including those who treat them. ${ }^{2}$ Standard recommendations for preventing the spread of infection are regular hand washing, covering coughs and sneezes, avoiding direct contact with livestock and wild animals, and avoiding close contact with anyone showing symptoms of respiratory illness such as coughing and sneezing. ${ }^{4}$ Furthermore, implementing Infection Prevention and Control (IPC) in healthcare facilities, especially in emergency units, operating rooms, clinics, and wards, is needed to reduce the spread of COVID-19. This study aimed to describe COVID-19 early detection and primary prevention system among staff and residents in the Obstetrics and Gynecology (OBSGYN) Department, Faculty of Medicine, Public Health, and Nursing, Universitas Gadjah Mada and Dr. Sardjito General Hospital. We hope that this study could become reference or be used for comparison in other departments in our hospital or obstetrics and gynecology departments in other hospitals.

\section{Method}

This research was a descriptive observational study. The data were acquired from the records of the COVID-19 taskforce in the OBSGYN Department. The study was conducted in Dr. Sardjito General Hospital, the primary education hospital for Obstetrics and Gynecology residents from the Faculty of Medicine, Public Health and Nursing, Universitas Gadjah Mada, from April to June 2020.

In order to describe the COVID-19 early detection and primary prevention system in OBSGYN Department, we looked for data from the regular reports of the COVID-19 taskforce in the department and then identified data that are relevant to the research, namely: the notes from taskforce periodic meetings, recapitulation of the data of suspected COVID-19 patients in OBSGYN Department, recapitulation of staff/residents who had close contact with suspected/confirmed COVID-19 patients, weekly reports from the COVID-19 taskforce, and department policies in schedule arrangement and protocol of Personal Protective Equipment (PPE) usage.

\section{Results}

Since the start of the pandemic, the OBSGYN department of Dr. Sardjito Hospital established a COVID-19 taskforce which consisted of representatives from staff and residents. Their duty is to formulate the main policy and organize the system of transmission prevention and early detection for specific use in the OBSGYN department. They also need to coordinate with the Infection Control and Prevention Committee of Dr. Sardjito Hospital in their efforts. The policy will be reviewed and approved by the head of the department and then it will be socialized to all the staff and residents. Based on the data acquisition phase, we found four main parameters of the COVID-19 early detection and primary prevention system in the OBSGYN Department, namely: a) Arrangement of staff and residents watch schedules; b) Usage of PPE according to the protocol; c) Contact tracing; and d) Early detection of COVID-19

\subsection{Arrangement of Staff and Residents Watch Schedules}

\subsubsection{Arrangement of Staff Watch Schedules}

The total number of consultants and specialist staff in our department is 27 (Table 1). The oldest staff is 81 years old while the youngest is 35 years old (mean age of $51.2 \pm 14.7$ years). Eighteen staff (67\%) are male and the rest (9 staff/33\%) are female. Seven staff $(26 \%)$ are $>60$ years old, which means they have the highest risk of contracting COVID-19 because of their age.

The arrangement of watch schedules for the staff was intended primarily for surgery on patients who had positive COVID-19 rapid antibody test or PCR test. The staff who are less than 60 years old ( $n$ 
= 18) were divided into 3 teams, each consisting of 6 staff. Each staff member was responsible for surgery related to COVID-19 patients for 1 day per week.

In addition, another policy from the department was that the staff who were $>60$ years old were only allowed to enter the green zone of the hospital (the zone which has the lowest risk of COVID-19 exposure). Our hospital also determined that elective surgery was temporarily postponed during the COVID-19 pandemic, therefore our department was only allowed to perform emergency obstetrics or gynecological surgery. The operating rooms for suspected/confirmed COVID-19 patients and nonCOVID-19 were also differentiated.

\subsubsection{Arrangement of Resident Watch Schedules}

The total number of active OBSGYN residents in Dr. Sardjito General Hospital is 74 (Table 1). The maximum age of the resident is 36 years old and the minimum age is 24 years old with a mean age of $31 \pm$ 2.5 years. Forty-one residents are male (54\%) and 33 residents are female (46\%).

Before the pandemics, besides working in Dr. Sardjito General Hospital, the residents were also sent to affiliated hospitals in weekly cycles. Several affiliated hospitals were located outside Yogyakarta. The residents had a higher risk of COVID-19 exposure because of higher patient load in the affiliated hospitals and from the weekly travel in and out of Yogyakarta. Therefore, the department decided to withdraw all of its residents from affiliated hospitals

Table 1. Characteristics of staff and residents in OBSGYN Department of Dr. Sardjito General Hospital

\begin{tabular}{lllll}
\hline Characteristic & $\begin{array}{l}\text { Staff } \\
(\mathbf{n = 2 7})\end{array}$ & \multicolumn{3}{l}{$\begin{array}{l}\text { Residents } \\
(\mathbf{n}=\mathbf{7 4 )}\end{array}$} \\
\cline { 2 - 5 } & $\mathbf{N}$ & $\mathbf{\%}$ & $\mathbf{N}$ & $\mathbf{\%}$ \\
\hline Gender & & & & \\
\hline Male & 18 & 67 & 41 & 54 \\
\hline Female & 9 & 33 & 33 & 46 \\
\hline Age (year) & & & & \\
\hline Max. & 81 & & 24 & \\
\hline Min. & 35 & & 36 & \\
\hline Mean \pm SD & $51.2 \pm 14.7$ & & $31 \pm 2.5$ & \\
\hline Age categories & & & & - \\
\hline$<60$ years old & 7 & 26 & - & - \\
\hline$\geq 60$ years old & 20 & 74 & - & \\
\hline
\end{tabular}

during the COVID-19 pandemic. All residents were concentrated in Dr. Sardjito General Hospital, the watch team in every unit and stage were downsized, and the schedule was arranged so that no resident would have 2 days watch in a row. Each shift for residents was 12 hours namely from 7 am to $7 \mathrm{pm}$ and from $7 \mathrm{pm}$ to $7 \mathrm{am}$. All of the residents had to use PPE in accordance with the provisions which are described next.

\subsubsection{Use of PPE according to the protocol}

The OBSGYN department had issued several regulations related to PPE, both for the staff and the residents. When arriving at the hospital, the staff and residents must immediately change their clothes into the prepared protective clothes. After that, the staff and residents used their PPE in accordance with the level in their respective zone. The PPE level referred to the Center for Disease Control and Prevention (CDC) international guidelines as well as the guidelines from the infection control team of Dr. Sardjito General Hospital.

The complete PPE protocol according to the workplace is as follows:

1. PPE Level 1

PPE level 1 consists of a scrub, cap/hat/head cover, surgical mask, and gown. PPE level 1 is used in areas with a low risk of COVID-19 transmission such as hallways and hospital areas that are not directly related to patients (Figure 1a).

2. PPE level 2

PPE level 2 consists of a scrub, cap/hat/head cover, surgical mask, face shield/goggle, gown, and gloves when doing a physical examination. PPE level 2 is used in areas with exposure to patients, such as wards, polyclinic, emergency departments, and delivery rooms. If the place has a higher risk of transmission, an N95 mask and boots can be added (Figure 1b).

\section{PPE level 3}

PPE level 3 consists of a scrub, cap/hat/head cover, surgical mask, N95 mask, face shield/ goggle, coverall jumpsuit, first glove (short), second glove (long), and boots. PPE level 3 is used in areas with the highest exposure to COVID-19 such as the COVID-19 isolation ward, specialized 
a.

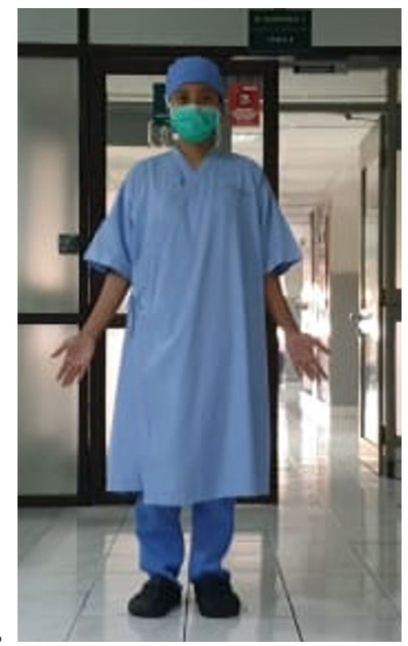

b.

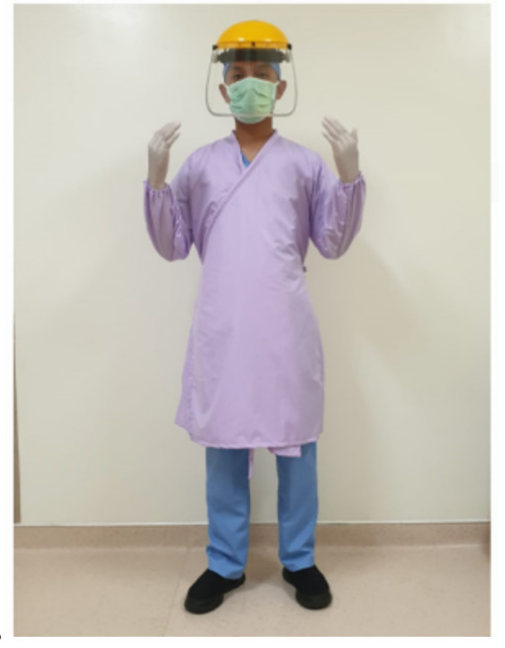

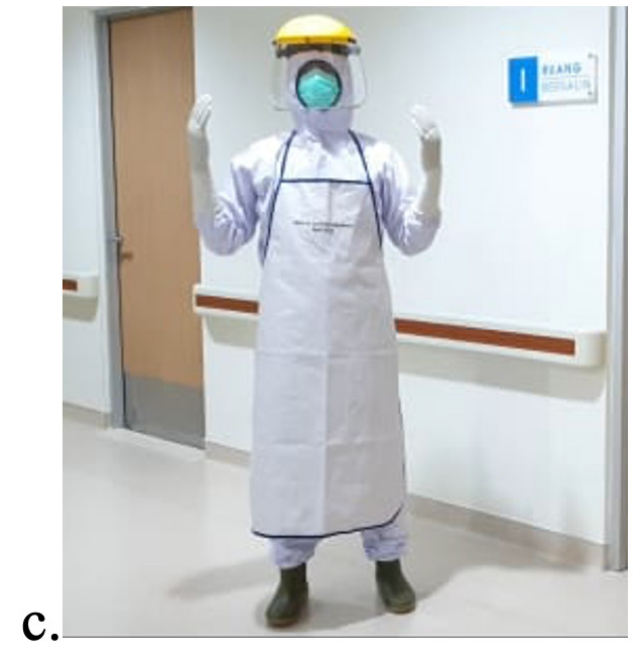

Figure 1a. PPE level 1; 1b. PPE level 2; and 1c. PPE level 3

delivery room and operation room for COVID-19 patients (Figure 1c).

The childbirth process with suspected or confirmed COVID-19 patients was done using a delivery chamber which was specially constructed according to the size of the patient's delivery bed.

In order to optimize the use of PPE properly and correctly, training on the use of PPE was conducted by the infection control team from Dr. Sardjito General Hospital, therefore the correct use of PPE could be well socialized to the staff and residents in OBSGYN Department. All PPE is stored in a special PPE room and excellent record keeping and documentation are done regarding the utilization of PPE. An inspection on the completeness and condition of all PPE is done every week.

\subsection{Contact tracing}

Contact tracing was conducted among the staff or residents who were exposed to patients with positive COVID-19 rapid antibody test or positive PCR test. It is conducted in a structured manner every day in accordance with the chronology of suspected or confirmed COVID-19 patients. A good record of the tracing is routinely made by the COVID-19 taskforce.

A total of 11 out of 27 (40\%) staff were exposed to suspected COVID-19 patients and 2 staff were selfisolated due to exposure to patients with positive COVID-19 (+) rapid antibody test. Meanwhile, a total of 65 out of 74 residents ( $88 \%$ ) had been exposed to suspected COVID-19 patients, and among the exposed residents, 12 residents (18\%) were self-isolated because of occurrence of clinical manifestations, exposure to patients with positive COVID-19 rapid antibody test, or exposure to confirmed COVID-19 patients based on PCR test.

\subsection{COVID-19 early detection}

Early detection of COVID-19 is done for the staff and residents by conducting rapid immunoglobulin $M$ (IgM) and immunoglobulin G (IgG) antibodies test. This test can be used as a regular screening test for health workers as well as the initial test in persons who are exposed to suspected or confirmed COVID-19 patients or persons who showed symptoms of COVID-19. The COVID-19 rapid antibody test is done using standardized reagents, namely using the Vazyme 2019-nCOVID IgM / IgG detection kit in collaboration with the COVID polyclinic of $\mathrm{Dr}$. Sardjito General Hospital and the Clinical Pathology Laboratory of Dr. Sardjito General Hospital.

As many as 13 of 27 staff (48\%) and 66 of 74 residents (89\%) have undergone rapid COVID-19 antibody test with $100 \%$ negative results. Furthermore, residents and staff who showed clinical manifestations, were exposed to patients with positive COVID-19 rapid antibody test, or were exposed to confirmed COVID-19 patients based 
from PCR test, could also report those events to the Occupational Safety and Health team of Dr. Sardjito General Hospital for a more comprehensive examination (physical examination, routine blood tests, and chest $\mathrm{x}$-ray) at the COVID-19 Polyclinic of Dr. Sardjito General Hospital. Until the end of the study, no staff member or resident has been positively confirmed to have COVID-19 infection.

\section{Discussion}

Numerous hospitals in Indonesia encourage their staff to postpone or reduce the number of elective surgeries and reduce the operating hours of polyclinics. However, in OBSGYN department, the rates of emergency surgery and spontaneous delivery were still high due to almost constant number of pregnancies in population. There are also many pregnant patients who are asymptomatic but actually have contracted the infection. Therefore, OBSGYN departments need to have a more stringent policy in COVID-19 prevention. This study is the first article to discuss the transmission prevention system and early detection of COVID-19 for obstetrics and gynecology staff and residents in Indonesia. There have been several national and international recommendations regarding transmission prevention and early detection of COVID-19.

The Indonesian Association of Obstetrics and Gynecology (POGI) issued several recommendations for health workers during the COVID-19 pandemic which include advice for healthcare personnel to immediately notify the personnel in charge of infection control and prevention in their workplace (ICP Committee) when there are pregnant women with confirmed COVID-19 status or Patients Under Supervision (PDP) status. This recommendation has been obliged by our department in which we collaborate with the ICP committee of Dr. Sardjito General Hospital in formulating the guidelines for management of pregnant patients with suspected/ confirmed COVID-19 status. In addition, rational, precise, and consistent use of PPE can reduce the risk of transmission of COVID-19. ${ }^{5}$ The proper use of PPE is related to proper supply and storage, training in regular use of PPE, and the strict discipline of every healthcare personnel. ${ }^{6,7}$
In addition, our department has also been following several recommendations from the World Health Organization (WHO). ${ }^{8}$ According to WHO recommendations for protection of health workers, residents and staff who are exposed to patients with positive COVID-19 rapid antibody test or PCR test are required to be self-isolated for 14 days with periodic temperature measurements and observations of respiratory symptoms. ${ }^{5}$ The recommended early detection test consists of rapid antibody test or rapid antigen test, while the gold standard is PCR testing using nasopharyngeal/oropharyngeal swab samples. ${ }^{8-10}$

In terms of patient management, it is also done according to WHO guidelines, namely guidelines for doctors who treat patients in the hospital to provide easy access and ensure the best management for patients. The guidelines include: 1) a triage process for identifying and sorting patients; 2) appropriate infection control precautions; 3) providing therapy and monitoring; 4) collection of specimens for laboratory diagnosis; 5) management of hypoxemic respiratory failure and acute respiratory distress syndrome; 6) septic shock management; 7) prevention of complications; 8) special treatment for COVID-19; and 9) specific recommendations for pregnant patients. ${ }^{5,11}$

Until now, there has been no study in Indonesia that specifically discusses the prevention of transmission and early detection of COVID-19 in the OBSGYN departments. Most of the efforts made in the OBSGYN department of Dr. Sardjito General Hospital is in accordance with the CDC and WHO recommendations but there are many points that can be improved. Other studies also described measures and policies to prevent transmission of COVID-19, such as the presence of a COVID-19 PCR swab facility for health workers with symptoms; a mandatory temperature measurement is done $2 x$ a day for health workers and pathway for health workers with a temperature $>37.5^{\circ} \mathrm{C}$ for further examination; and implementation of the rule which state that each health worker is only allowed to work at one institution. ${ }^{12-14}$ In the future, those measures should be reviewed and determined whether they could also be implemented in order to strengthen COVID-19 prevention system in our department. 
Further study regarding other contingency measures are also needed to be conducted in order to anticipate the dynamic nature of pandemics, such as when the rate of infection is increasing rapidly or the reverse and when the effective treatments or vaccines are found.

\section{Conclusion}

This study is the first article to discuss the transmission prevention system and early detection of COVID-19 for obstetrics and gynecology staff and residents in Indonesia. Several efforts to prevent the transmission of COVID-19 have been conducted by the department in accordance with national and international recommendations, namely arrangement of watch schedule, usage of PPE, contact tracing, and the early detection system. Until the end of the study, no staff or resident has been positively confirmed to have COVID-19 infection.

\section{Conflict of interests}

There is no conflict of interest

\section{References}

1. World Health Organization. Coronavirus 2020 [cited 2020, August 27]. Available from: https://www.who.int/health-topics/ coronavirus\#tab=tab_1.

2. World Health Organization. COVID-19 Situation update for the WHO African Region: External Situation Report 4. 2020;1-7 2020 [cited 2020, September 18]. Available from: https://apps. who.int/iris/bitstream/handle/10665/331587/ SITREP_COVID-19_WHOAFRO_20200325-eng. pdf.

3. Karyono DR, Wicaksana AL. Current prevalence, characteristics, and comorbidities of patients with COVID-19 in Indonesia. J Community Empowerment Health. 2020;3(2):8.

4. Centers for Disease Control and Prevention. How to Protect Yourself \& Others 2020 [cited 2020, November 4]. Available from: https://www.cdc. gov/coronavirus/2019-ncov/prevent-gettingsick/prevention.html.

5. World Health Organization. Clinical management of severe acute respiratory infection when novel coronavirus (2019-nCoV) infection is suspected: interim guidance, 28 January 2020. Geneva: World Health Organization, 2020 2020. Report No.: Contract No.: WHO/nCoV/Clinical/2020.3.

6. World Health Organization. WHO DirectorGeneral's Remarks at the Media Briefing on 2019-nCoV on 11 February 2020 2020. Available from: https://www.who.int/director-general/ speeches/detail/who-director-general-sremarks-at-the-media-briefing-on-2019-ncovon-11-february-2020.

7. Wee LE, Sim XYJ, Conceicao EP, Aung MK, Goh JQ, Yeo DWT, et al. Containment of COVID-19 cases among healthcare workers: the role of surveillance, early detection, and outbreak management. Infection Control and Hospital Epidemiology. 2020;41(7):765-71.

8. Perhimpunan Dokter Spesialis Patologi Klinik dan Kedokteran Laboratorium Indonesia. Panduan Tatalaksana Pemeriksaan Rapid Tes Antibody Sars-Cov-2 Metode Imunokromatografi. 2020.

9. Kementerian Kesehatan Republik Indonesia. Pedoman Kesiapsiagaan Menghadapi Coronavirus Disease (COVID-19) Revisi ke3. Jakarta: Kementerian Kesehatan Republik Indonesia; 2020.

10. Setiati S, Azwar M. COVID-19 and Indonesia. Acta Medica Indonesiana. 2020;52:84-9.

11. Djasri H. Corona virus dan manajemen mutu pelayanan klinis di rumah sakit. Journal of Hospital Accreditation. 2020;2(1):2.

12. Gan WH, Lim JW, Koh D. Preventing intra-hospital infection and transmission of Coronavirus Disease 2019 (COVID-19) in health-care workers. Safety and Health at Work. 2020;11(2):241-3.

13. Yang P, Wang X. COVID-19: a new challenge for human beings. Cellular \& Molecular Immunology. 2020;17(5):555-7.

14. Black JRM, Bailey C, Przewrocka J, Dijkstra KK, Swanton C. COVID-19: the case for healthcare worker screening to prevent hospital transmission. Lancet. 2020;395(10234):141820. 